\title{
Sarcopenia is not associated with inspiratory muscle strength but with expiratory muscle strength among older adults requiring long-term care/support
}

\author{
Yohei Sawaya ${ }^{\text {Corresp., Equal first author, } 1,2}$, Takahiro Shiba ${ }^{\text {Equal first author, } 2}{ }^{2}$, Masahiro Ishizaka ${ }^{1}$, Tamaki Hirose ${ }^{1,2}$, Ryo Sato ${ }^{2}$, \\ Akira Kubo ${ }^{1}$, Tomohiko Urano \\ Corresp. 2,3 \\ 1 Department of Physical Therapy, School of Health Sciences, International University of Health and Welfare, Otawara, Tochigi, Japan \\ 2 Nishinasuno General Home Care Center, Department of Day Rehabilitation, Care Facility for the Elderly “Maronie-en”, Nasushiobara, Tochigi, Japan \\ 3 Department of Geriatric Medicine, School of Medicine, International University of Health and Welfare, Narita, Chiba, Japan \\ Corresponding Authors: Yohei Sawaya, Tomohiko Urano \\ Email address: sawaya@iuhw.ac.jp, turano@iuhw.ac.jp
}

Background. Recently, the concept of respiratory sarcopenia has been advocated, but evidence is lacking regarding which respiratory parameters are appropriate indicators. Therefore, we investigated the association between sarcopenia, respiratory function, and respiratory muscle strength to identify the most appropriate parameters for respiratory sarcopenia. Methods. We included 124 older adults (67 men, 57 women; average age $77.2 \pm 8.3$ years) requiring long-term care/support who underwent Day Care for rehabilitation. Handgrip strength, usual gait speed, and skeletal muscle mass were measured using bioelectrical impedance analysis. Participants were then diagnosed with sarcopenia using the algorithm of the Asian Working Group for Sarcopenia 2019. Parameters of respiratory function (forced vital capacity, forced expiratory volume in one second [FEV1.0], FEV1.0\%, and peak expiratory flow rate) and respiratory muscle strength (maximal expiratory pressure [MEP] and maximal inspiratory pressure) were also measured according to American Thoracic Society guidelines. Respiratory parameters significantly related to sarcopenia were identified using binomial logistic regression and receiver operating characteristic analyses. Results. Seventy-seven participants were classified as having sarcopenia. Binomial logistic regression analysis showed that MEP was the only respiratory parameter significantly associated with sarcopenia. The cut-off MEP value for predicting sarcopenia was $47.0 \mathrm{cmH}_{2} 0$ for men and $40.9 \mathrm{cmH}_{2} 0$ for women.

Conclusions. The most appropriate parameter for assessing respiratory sarcopenia may be MEP, which is an indicator of expiratory muscle strength, rather than FVC, MIP, or PEFR, as suggested in previous studies. Measuring MEP is simpler than measuring respiratory function parameters. Moreover, it is expected to have clinical applications such as respiratory sarcopenia screening . 


\section{Sarcopenia is not associated with inspiratory muscle}

2 strength but with expiratory muscle strength among

3 older adults requiring long-term care/support

4

Yohei Sawaya, Equal first author, $1,2^{*}$, Takahiro Shiba, ${ }^{\text {Equal first author, }, 2}$, Masahiro Ishizaka ${ }^{1}$, Tamaki Hirose $^{1,2}$, Ryo Sato ${ }^{2}$, Akira Kubo ${ }^{1}$, and Tomohiko Urano ${ }^{2,3^{*}}$

${ }^{1}$ Department of Physical Therapy, School of Health Sciences, International University of Health and Welfare, Tochigi, Japan

${ }^{2}$ Nishinasuno General Home Care Center, Department of Day Rehabilitation, Care Facility for the Elderly "Maronie-en," Tochigi, Japan

${ }^{3}$ Department of Geriatric Medicine, School of Medicine, International University of Health and Welfare, Chiba, Japan

\section{*Corresponding author}

Tomohiko Urano, $\mathrm{MD}, \mathrm{PhD}$

Department of Geriatric Medicine, School of Medicine, International University of Health and Welfare: 4-3 Kozunomori, Narita city, Chiba, 286-8686, Japan

Email: turano@iuhw.ac.jp

TEL: +81-287-36-6622

FAX: +81-287-36-8510

Yohei Sawaya, RPT, PhD

Department of Physical Therapy, School of Health Sciences, International University of Health and Welfare: 2600-1 Kitakanemaru, Otawara city, Tochigi 324-8501, Japan

Email: sawaya@iuhw.ac.jp

TEL: +81-287-24-3018

Fax: $+81-287-24-3100$

\section{Abstract}

Background. Recently, the concept of respiratory sarcopenia has been advocated, but evidence is lacking regarding which respiratory parameters are appropriate indicators. Therefore, we investigated the association between sarcopenia, respiratory function, and respiratory muscle strength to identify the most appropriate parameters for respiratory sarcopenia.

Methods. We included 124 older adults (67 men, 57 women; average age $77.2 \pm 8.3$ years) requiring long-term care/support who underwent Day Care for rehabilitation. Handgrip strength, usual gait speed, and skeletal muscle mass were measured using bioelectrical impedance analysis. Participants were then diagnosed with sarcopenia using the algorithm of the Asian 
40 Working Group for Sarcopenia 2019. Parameters of respiratory function (forced vital capacity,

41 forced expiratory volume in one second [FEV1.0], FEV1.0\%, and peak expiratory flow rate) and

42 respiratory muscle strength (maximal expiratory pressure [MEP] and maximal inspiratory

43 pressure) were also measured according to American Thoracic Society guidelines. Respiratory

44 parameters significantly related to sarcopenia were identified using binomial logistic regression

45 and receiver operating characteristic analyses.

Results. Seventy-seven participants were classified as having sarcopenia. Binomial logistic regression analysis showed that MEP was the only respiratory parameter significantly associated with sarcopenia. The cut-off MEP value for predicting sarcopenia was $47.0 \mathrm{cmH}_{2} 0$ for men and $40.9 \mathrm{cmH}_{2} \mathrm{O}$ for women.

Conclusions. The most appropriate parameter for assessing respiratory sarcopenia may be MEP, which is an indicator of expiratory muscle strength, rather than FVC, MIP, or PEFR, as suggested in previous studies. Measuring MEP is simpler than measuring respiratory function parameters. Moreover, it is expected to have clinical applications such as respiratory sarcopenia screening.

57

Keywords: Elderly; Respiratory muscles; Respiratory sarcopenia; Spirometry; Maximal expiratory pressure

\section{Introduction}

Respiratory sarcopenia has been recently defined as "whole-body sarcopenia, and low respiratory muscle mass followed by low respiratory muscle strength and/or deteriorated respiratory function" (Nagano et al., 2021). However, it is difficult to assess low respiratory muscle mass as no cut-off value for it has been established (Nagano et al., 2021). Therefore, criteria other than respiratory muscle mass are used to diagnose respiratory sarcopenia (Nagano et al., 2021). The indicators used to assess respiratory sarcopenia were maximal inspiratory pressure (MIP) and forced vital capacity (FVC). However, Kera et al., who were the first to define respiratory sarcopenia, used peak expiratory flow rate (PEFR) as a respiratory parameter for evaluating respiratory sarcopenia (Kera et al., 2019). Several other respiratory parameters have been reported to be associated with sarcopenia, but it is unclear which one is the most appropriate for respiratory sarcopenia (Ohara et al., 2018; Ohara et al., 2020; Ridwan et al., 2021; Mishra et al., 2020). The review also clearly stated that "there is a lack of evidence in the specificity of the measures and its cut-off values" (Nagano et al., 2021). Thus, further accumulation of evidence is essential.

Respiratory parameters are divided into those used to assess respiratory function, such as FVC and PEFR, or respiratory muscle strength. Respiratory muscle strength is considered to be the total muscle strength of the diaphragm and other respiratory muscles and is defined as the maximal expiratory pressure (MEP) and MIP (Cook, Mead \& Orzalesi, 1964). However, no 
80

81

82

83

84

85

86

87

88

89

90

91

92

93

94

95

96

97

98

99

100

101

102

103

104

105

106

107

108

109

110

111

112

113

114

115

116

117

118

119

120

121

122

study has simultaneously analyzed the association between sarcopenia, respiratory function, and respiratory muscle strength.

Regarding the respiratory muscles, the main inspiratory muscle is the diaphragm (De Troyer \& Estenne, 1988; Epstein, 1994). The diaphragm differs from skeletal muscles considering its special embryological characteristics and its ability to continuously contract and relax (Jinguji, 1978; Fujishima et al., 2019). The muscles of deglutition, which show similar characteristics, are thought to be less prone to muscle atrophy than skeletal muscles, and this difference should be considered regarding sarcopenia for these muscles (Fujishima et al., 2019). In addition, the diaphragm has a high percentage of fatigue-resistant fibers (Epstein, 1994). Based on the above, we hypothesized that the diaphragm might be less likely to develop respiratory sarcopenia. Furthermore, because our prior fundamental study showed that MEP was independently associated with skeletal muscle mass index (SMI), we also hypothesized that sarcopenia is more associated with expiratory muscle strength than inspiratory muscle strength (Sawaya et al., 2020).

Therefore, this study aimed to determine the most appropriate parameter for assessing respiratory sarcopenia by measuring both respiratory function and respiratory muscle strength. Moreover, we aimed to calculate the cut-off value for the obtained parameter.

\section{Materials \& Methods Study design}

This was a cross-sectional study conducted at a single daycare between March 2018 and August 2019. All participants were informed of the study orally and in writing, and written consent was obtained from all participants. The Ethical Review Committee of the International University of Health and Welfare approved this study (Approval No.: 17-Io-189-7). The study complied with the principles of the Declaration of Helsinki.

\section{Study participants}

This study included 154 community-dwelling older adults aged 60 years or older who were under Day Care. All participants had been certified as requiring long-term care/support among the Japanese system (Yamada \& Arai, 2020). Our Day Care for older adults involves rehabilitation services that include exercise, transportation, meals, and bathing options. The study included those whose body composition parameters could be measured in the standing position. We excluded those who had been diagnosed as having dementia or aphasia, those for whom performing spirometry was difficult, and those with respiratory diseases such as those on home oxygen therapy or with a forced expiratory volume \% in 1 second (FEV1.0\%) of $<70 \%$, considering the effect of airway obstruction on respiratory function according to a previous study (Kera et al., 2019).

\section{Sarcopenia assessment}

Sarcopenia was diagnosed based on the algorithm of the Asian Working Group for Sarcopenia (AWGS) 2019 (Chen et al., 2020), which was characterized by low skeletal muscle mass, low muscle strength, and/or low physical function. Grip strength was measured twice (Smedley-type hand dynamometer, TKK 5401 Grip-D, Takei Scientific Instruments, Japan) on each side in the 
123 sitting position, and the maximum value was used as the representative value. Usual gait speed

124 was measured once at a distance of $5 \mathrm{~m}$ using a stopwatch (Arai, 2018). In addition to the

125 measurement section of $5 \mathrm{~m}$, acceleration and deceleration paths were set as walking paths.

126 Skeletal and trunk muscle mass were measured using a multifrequency bioelectrical impedance

127 analysis (BIA) body composition analyzer (InBody 520, InBody, Japan), and the skeletal muscle

128 mass index (SMI) was calculated by dividing the skeletal muscle mass of limbs by the square of

129 the height. The cutoff for each measurement is as follows: grip strength $<28.0 \mathrm{~kg}$ for men and $<$

$13018.0 \mathrm{~kg}$ for women, usual gait speed $<1.0 \mathrm{~m} / \mathrm{sec}$ for both men and for women, and SMI $<7.0$

$131 \mathrm{~kg} / \mathrm{m}^{2}$ for men and $<5.7 \mathrm{~kg} / \mathrm{m}^{2}$ for women (Chen et al., 2020).

132

133

\section{Respiratory function and muscle strength}

134 Respiratory function and muscle strength were measured using a spirometer (Autospiro AS-507,

135 Minato, Japan) and an attached unit (AAM377, Minato, Japan). All measurements were

136 performed by physical therapists based on the American Thoracic Society (ATS)/European

137 Respiratory Society (ERS) guidelines (American Thoracic Society/European Respiratory

138 Society, 2002). Respiratory function mode involved FVC, forced expiratory volume in one

139 second (FEV1.0), FEV1.0\%, and PEFR. The respiratory muscle strength mode involved MEP

140 and MIP. Respiratory function was measured first, followed by respiratory muscle strength. The

141 maximum value after three measurements was used as the representative value.

142

\section{Basic attributes}

144 Details regarding age, height, care level, smoking history, and morbidity were collected from the medical records of the Day Care. Since long-term certification is classified into seven care levels, this study used an ordinal scale, with a ranking from 1 (mild) to 7 (severe) (Yamada \& Arai, 2020). Body weight was obtained from the data of body composition measurement.

\section{Statistical analyses}

First, the basic attributes and measurement values of the sarcopenia and non-sarcopenia groups were compared using the unpaired t-test, Wilcoxon rank-sum test, chi-square test, and Fisher's exact test. Second, binomial logistic regression analysis using stepwise selection was conducted with the presence or absence of sarcopenia as the dependent variable. Respiratory parameters, after considering multicollinearity, were considered as independent variables. Sex, age, care level, body mass index, and morbidity were used as control variables. Third, for the respiratory parameters extracted by binomial logistic regression analysis, the area under the curve (AUC), sensitivity, and specificity were calculated using the Youden Index method from the receiver operating characteristic (ROC) curve, and the cut-off value of sarcopenia was determined. Fourth, the MEP and MIP components were analyzed using partial correlation, controlling for sex and age. The partial correlation was performed after confirming the normal distribution of trunk muscle mass per height squared and SMI by Kolmogorov-Smirnov test and Shapiro-Wilk test. Statistical analysis was performed using SPSS version 25 (IBM Japan, Japan), with a significance level of 5\%. Power analysis was performed using G*Power version 3.1.9.2 (Faul et al., 2007). 


\section{Results}

167

168

169

170

171

172

173

174

175

176

177

178

179

180

181

182

183

184

185

186

187

188

189

190

191

192

193

194

195

196

197

198

199

200

201

202

203

Figure 1 shows the flowchart of the study participants. After applying the exclusion criteria, there were 124 eligible participants, 77 with sarcopenia and 47 with non-sarcopenia. Table 1 shows a comparison of the basic attributes and measurement values between the sarcopenia and non-sarcopenia groups. The sarcopenia group had a significantly lower MEP for men and PEFR and MEP for women. There was no significant difference in morbidity between the sarcopenia and non-sarcopenia groups.

Insert Figure 1 about here.

Insert Table 1 about here.

Binomial logistic regression analysis showed that MEP was the only respiratory parameter that was significantly associated with sarcopenia (Table 2). Four parameters (FVC, FEV1.0\%, PEFR, and MIP) were excluded from the independent variables by the stepwise selection method. FEV1.0 was excluded after multicollinearity was considered. Post-hoc power analysis was performed for binomial logistic regression analysis. For the multiple linear regression of $\mathrm{F}$ tests, the power was 0.99 , based on the effect size of 0.39 , calculated from the regression equation (Nagelkerke $\mathrm{R}^{2}=0.281$ ). Analyses by sex are shown in Table $\mathrm{S} 1$ and $\mathrm{S} 2$. Figure 2 shows ROC curves. The cut-off value of MEP for predicting sarcopenia was $47.0 \mathrm{cmH}_{2} 0$ in men (AUC 0.689 , sensitivity $91.7 \%$, specificity $41.9 \%, \mathrm{p}=0.011$ ) and $40.9 \mathrm{cmH}_{2} \mathrm{O}$ in women (AUC 0.677, sensitivity $65.2 \%$, specificity $70.6 \%, \mathrm{p}=0.024)$.

Insert Table 2 about here.

Insert Figure 2 about here.

Table 3 shows the results of the partial correlation analysis. MEP and MIP showed a significant positive correlation $(\mathrm{r}=0.550)$ with each other. MEP also showed a significant positive correlation with grip strength, usual gait speed, and SMI, while MIP was not significantly associated with these parameters.

Insert Table 3 about here.

\section{Discussion}

In previous reports on sarcopenia and respiratory parameters, skeletal muscle mass was calculated from predictive equations (Ohara et al., 2018; Ohara et al., 2020; Ridwan et al., 2021), and original cut-off values were used for grip strength, walking speed, and SMI for sarcopenia (Kera et al., 2019). This study is the first report to combine (1) compliance with the AWGS2019 criteria, (2) skeletal muscle mass measurement using the BIA method recommended by AWGS and EWGSOP (Chen et al., 2020; Cruz-Jentoft et al., 2019), (3) simultaneous analysis of respiratory function and respiratory muscle strength, and (4) compliance with ATS guidelines for spirometry instruments (American Thoracic Society/European Respiratory Society, 2002). Our results showed the lack of significant association between sarcopenia and MIP and FVC as indicators of respiratory sarcopenia and a significant association between sarcopenia and MEP. 
204 These findings may be explained by muscle atrophy of the diaphragm and rectus abdominis as

205

206

207

208

209

210

211

212

213

214

215

216

217

218

219

220

221

222

223

224

225

226

227

228

229

230

231

232

233

234

235

236

237

238

239

240

241

242

243

well as the component factors of MEP.

Sarcopenia was not associated with inspiratory muscle strength of inspiration but with expiratory muscle strength. Muscle fibers are classified into type 1 fibers (slow-oxidative and fatigue-resistant), type 2A fibers (fast-oxidative-glycolytic and fatigue-resistant), and type 2B fibers (fast-glycolytic and fatigue-sensitive) (Epstein, 1994). The diaphragm, which is the main inspiratory muscle, is composed of $80 \%$ fatigue-resistant fibers (55\% type 1,25\% type $2 \mathrm{~A}$ ), while the rectus abdominis, the main expiratory muscle, is composed of $46 \%$ type $2 \mathrm{~B}$ fibers (Epstein, 1994; Ito, 1998). In sarcopenia, muscle atrophy is selectively observed in fast-twitch fibers (Lexell, Taylor \& Sjöström, 1988), suggesting that the diaphragm may be less atrophic and maintain its inspiratory muscle strength, while the rectus abdominis may be more prone to atrophy and loss of expiratory muscle strength. These supportive reports indicate that the diaphragm shows very little change in muscle mass and strength with aging (Krumpe et al., 1985; Caskey et al., 1989; Polkey et al., 1997; Mizuno, 1991). Although a study reported that the diaphragm is significantly thinner in older adults with sarcopenia, it is not clear whether the change is clinically significant because only a difference of $0.2-0.4 \mathrm{~mm}$ was noted (Deniz et al., 2021).

Next, we performed a partial correlation analysis, focusing on the component factors of MEP and MIP. Although the MEP and MIP of the participants in this study were correlated, only MEP showed a significant association with grip strength, walking speed, and SMI, which are indicators of sarcopenia. Trunk muscle mass was also found to have a significant association only with MEP. These results suggest that the component factors of MEP and MIP are different, and that MEP may contain sarcopenia elements, while MIP does not. However, the association between these sarcopenia parameters and MEP/MIP is inconsistent across research populations (Shin et al., 2017; Bahat et al., 2014); thus, further research is needed. For the remaining respiratory function parameters, binomial logistic regression analysis did not show a significant association with sarcopenia in the overall and gender-specific analyses.

Our results showed that the cut-off value of MEP for predicting sarcopenia was 47.0 $\mathrm{cmH}_{2} \mathrm{O}$ for men and $40.9 \mathrm{cmH}_{2} 0$ for women. Only one previous study with a Brazilian population calculated a similar cut-off value for MEP, which was $60.0 \mathrm{cmH}_{2} 0$ for men and $50.0 \mathrm{cmH}_{2} 0$ for women (Ohara et al., 2018). Our study demonstrates acceptable results because the respiratory muscle strength of Asian ethnic groups is low owing to their small body size (Chen \& Kuo, 1989). FVC and PEFR, which are parameters of respiratory sarcopenia in previous studies, may be difficult to obtain for older adults even if they have no cognitive problems, because obtaining these parameters requires several repetitive breaths of varying intensity (Nagano et al., 2021; Kera et al., 2019). However, MEP only requires maximal expiratory effort from the maximal inspiratory position, which has the advantage of being easy to obtain.

This study has several limitations. Our participants were older adults requiring long-term care/support and may have been affected by disease conditions. However, it is difficult to determine whether sarcopenia and respiration are affected by primary (aging) or secondary

Peerj reviewing PDF | (2021:09:65876:3:0:NEW 6 Jan 2022) 
244 (disease, etc.) factors. For this reason, we included them as control variables in the binomial 245 logistic regression analysis. Since the multimorbidity of older Japanese adults was $62.8 \%$, we 246 could not eliminate disease-related factors (Aoki et al., 2018). The fact that the population had a 247 high sarcopenia prevalence and a low AUC value, the generalizability of this study requires 248 further survey among healthy individuals. Although MEP was found to predict whole-body 249 sarcopenia in this study, these findings do not indicate that MEP is associated with future 250 sarcopenic respiratory disability. Future longitudinal studies with a large sample size are needed 251 to clarify the clinical significance of each parameter in respiratory sarcopenia.

252

253

\section{Conclusions}

254 The most appropriate parameter for assessing respiratory sarcopenia may be MEP, which is an indicator of expiratory muscle strength, instead of FVC, MIP, or PEFR, as suggested in previous studies. It easy to instruct patients for MEP measurement. MEP is expected to be applied in clinical practice.

258

259

\section{Acknowledgments}

260

261

262

263

264

265

266

267

268

269

270

271

272

273

274

275

276

277

278

279

280

281

282

\section{References}

American Thoracic Society/European Respiratory Society. 2002. ATS/ERS Statement on respiratory muscle testing. American Journal of Respiratory Critical Care Medicine, 166(4):518-624. DOI: $10.1164 / \mathrm{rccm} .166 .4 .518$.

Aoki T, Yamamoto Y, Ikenoue T, Onishi Y, Fukuhara S. 2018. Multimorbidity patterns in relation to polypharmacy and dosage frequency: A nationwide, cross-sectional study in a Japanese population. Scientific Reports 8(1):3806. DOI: 10.1038/s41598-018-21917-6.

Arai H. Preface. 2018. Geriatric \& Gerontology International 18(S1): 5-6. DOI: 10.1111/ggi.13310.

Bahat G, Tufan A, Ozkaya H, Tufan F, Akpinar TS, Akin S, Bahat Z, Kaya Z, Kiyan E, Erten N, Karan MA. 2014. Relation between hand grip strength, respiratory muscle strength and spirometric measures in male nursing home residents. The Aging Male 17(3):136-140. DOI: $10.3109 / 13685538.2014 .936001$.

Caskey CI, Zerhouni EA, Fishman EK, Rahmouni AD. 1989. Aging of the diaphragm: A CT study. Radiology 171(2): 385-389. DOI: 10.1148/radiology.171.2.2704802.

Cook CD, Mead J, Orzalesi MM. 1964. Static volume-pressure characteristics of respiratory system during maximal efforts. Journal of Applied Physiology 19:1016-1022. DOI: 10.1152/jappl.1964.19.5.1016.

Chen HI, Kuo CS. 1989. Relationship between respiratory muscle function and age, sex, and other factors. Journal of Applied Physiology 66(2):943-948. DOI: 10.1152/jappl.1989.66.2.943. 
283

284

285

286

287

288

289

290

291

292

293

294

295

296

297

298

299

300

301

302

303

304

305

306

307

308

309

310

311

312

313

314

315

316

317

318

319

320

321

322

Chen LK, Woo J, Assantachai P, Auyeung TW, Chou MY, Iijima K, Jang HC, Kang L, Kim M, Kim S, Kojima T, Kuzuya M, Lee JSW, Lee SY, Lee WJ, Lee Y, Liang CK, Lim JY, Lim WS, Peng LN, Sugimoto K, Tanaka T, Won CW, Yamada M, Zhang T, Akishita M, Arai H. 2020. Asian working group for sarcopenia: 2019 consensus update on sarcopenia diagnosis and treatment. Journal of the American Medical Directors Association 21(3):300-307.e2. DOI: 10.1016/j.jamda.2019.12.012.

Cruz-Jentoft AJ, Bahat G, Bauer J, Boirie Y, Bruyère O, Cederholm T, Cooper C, Landi F, Rolland Y, Sayer AA, Schneider SM, Sieber CC, Topinkova E, Vandewoude M, Visser M, Zamboni M. 2019. Sarcopenia: Revised European consensus on definition and diagnosis. Age and Ageing 48(1):16-31. DOI: 10.1093/ageing/afy169.

De Troyer A, Estenne M. 1988. Functional anatomy of the respiratory muscles. Clinics in Chest Medicine 9(2):175-193.

Deniz O, Coteli S, Karatoprak NB, Pence MC, Varan HD, Kizilarslanoglu MC, Oktar SO, Goker B. 2021. Diaphragmatic muscle thickness in older people with and without sarcopenia. Aging Clinical and Experimental Research 33(3):573-580. DOI: 10.1007/s40520-02001565-5.

Epstein SK. 1994. An overview of respiratory muscle function. Clinics in Chest Medicine 15(4):619-639.

Faul F, Erdfelder E, Lang AG, Buchner A. 2007. G*Power 3: A flexible statistical power analysis program for the social, behavioral, and biomedical sciences. Behavior Research Methods 39(2):175-191. DOI: 10.3758/bf03193146.

Fujishima I, Fujiu-Kurachi M, Arai H, Hyodo M, Kagaya H, Maeda K, Mori T, Nishioka S, Oshima F, Ogawa S, Ueda K, Umezaki T, Wakabayashi H, Yamawaki M, Yoshimura Y. 2019. Sarcopenia and dysphagia: Position paper by four professional organizations. Geriatric \& Gerontology International 19(2):91-97. DOI: 10.1111/ggi.13591.

Ito J. 1998. Fiber type composition of abdominal muscles in Japanese macaques (Macaca fuscata). Okajimas Folia Anatomica Japan 74(6):199-205 DOI: 10.2535/ofaj1936.74.6_199.

Jinguji Y. 1978. Development of diaphragm in mouse embryos. Kitakanto Medical Journal 28(3):185-209. [In Japanese, English Abstract]. DOI: 10.2974/kmj1951.28.185.

Kera T, Kawai H, Hirano H, Kojima M, Watanabe Y, Motokawa K, Fujiwara Y, Ihara K, Kim H, Obuchi S. 2019. Definition of respiratory sarcopenia with peak expiratory flow rate. Journal of the American Medical Directors Association 20(8):1021-1025. DOI: 10.1016/j.jamda.2018.12.013.

Krumpe PE, Knudson RJ, Parsons G, Reiser K. 1985. The aging respiratory system. Clinics in Geriatric Medicine 1(1):143-175.

Lexell J, Taylor CC, Sjöström M. 1988. What is the cause of the ageing atrophy? Total number, size and proportion of different fiber types studied in whole vastus lateralis muscle from 15- to 83-year-old men. Journal of the Neurological Sciences 84(2-3):275-294. DOI: 10.1016/0022-510x(88)90132-3. 
323

324

325

326

327

328

329

330

331

332

333

334

335

336

337

338

339

340

341

342

343

344

345

346

347

348

349

350

351

352

353

354

355

356

Mishra A, Bigam KD, Extermann M, Faramand R, Thomas K, Pidala JA, Baracos VE. 2020. Sarcopenia and low muscle radiodensity associate with impaired $\mathrm{FEV}_{1}$ in allogeneic haematopoietic stem cell transplant recipients. Journal of Cachexia, Sarcopenia and Muscle 11(6):1570-1579. DOI: $10.1002 / \mathrm{jcsm} .12604$.

Mizuno M. 1991. Human respiratory muscles: Fibre morphology and capillary supply. The European Respiratory Journal 4(5):587-601.

Nagano A, Wakabayashi H, Maeda K, Kokura Y, Miyazaki S, Mori T, Fujiwara D. 2021. Respiratory sarcopenia and sarcopenic respiratory disability: Concepts, diagnosis, and treatment. The Journal of Nutrition, Health \& Aging 25(4):507-515. DOI: 10.1007/s12603-021-1587-5.

Ohara DG, Pegorari MS, Oliveira Dos Santos NL, de Fátima Ribeiro Silva C, Monteiro RL, Matos AP, Jamami M. 2018. Respiratory muscle strength as a discriminator of sarcopenia in community-dwelling elderly: A cross-sectional study. The Journal of Nutrition, Health \& Aging 22(8):952-958. DOI: $10.1007 / \mathrm{s} 12603-018-1079-4$.

Ohara DG, Pegorari MS, Oliveira Dos Santos NL, de Fátima Ribeiro Silva C, Oliveira MSR, Matos AP, Jamami M. 2020. Cross-sectional study on the association between pulmonary function and sarcopenia in Brazilian community-dwelling elderly from the Amazon region. The Journal of Nutrition, Health \& Aging 24(2):181-187. DOI: 10.1007/s12603019-1290-y.

Polkey MI, Harris ML, Hughes PD, Hamnegärd CH, Lyons D, Green M, Moxham J. 1997. The contractile properties of the elderly human diaphragm. American Journal of Respiratory and Critical Care Medicine 155(5):1560-1564. DOI: 10.1164/ajrccm.155.5.9154857.

Ridwan ES, Wiratama BS, Lin MY, Hou WH, Liu MF, Chen CM, Hadi H, Tan MP, Tsai PS. 2021. Peak expiratory flow rate and sarcopenia risk in older Indonesian people: A nationwide survey. PLoS One 16(2):e0246179. DOI: 10.1371/journal.pone.0246179.

Sawaya Y, Ishizaka M, Kubo A, Shiba T, Hirose T, Onoda K, Maruyama H, Urano T. 2020. Association between skeletal muscle mass index and lung function/respiratory muscle strength in older adults requiring long-term care or support. Journal of Physical Therapy Science 32(11):754-759. DOI: 10.1589/jpts.32.754.

Shin HI, Kim DK, Seo KM, Kang SH, Lee SY, Son S. 2017. Relation between respiratory muscle strength and skeletal muscle mass and hand grip strength in the healthy elderly. Annals of Rehabilitation Medicine 41(4):686-692. DOI: 10.5535/arm.2017.41.4.686.

Yamada M, Arai H. 2020. Long-term care system in Japan. Annals of Geriatric Medicine and Research 24(3):174-180. DOI: 10.4235/agmr.20.0037. 


\section{Table $\mathbf{1}$ (on next page)}

Table 1. Basic attributes and measurements values with and without sarcopenia

${ }^{* P}<0.05$ for unpaired t test. ${ }^{* * \mathrm{P}}<0.05$ for Wilcoxon rank-sum test. ${ }^{\dagger}$ Median $(25$ th percentile-75th percentile). "With missing data (63 men, 55 women). BMI, body mass index; SMI, skeletal muscle mass index; FVC, forced vital capacity; FEV1.0, forced expiratory volume in 1 second; PEFR, peak expiratory flow rate; MEP, maximal expiratory pressure; MIP, maximal inspiratory pressure; dis, disease. 
1 Table 1. Basic attributes and measurements values with and without sarcopenia

\begin{tabular}{|c|c|c|c|c|c|c|}
\hline & \multicolumn{3}{|c|}{$\operatorname{Men}(n=67)$} & \multicolumn{3}{|c|}{ Women $(n=57)$} \\
\hline & $\begin{array}{l}\text { Sarcopenia } \\
\quad(n=43)\end{array}$ & $\begin{array}{c}\text { Non- } \\
\text { sarcopenia } \\
(\mathrm{n}=24)\end{array}$ & P-value & $\begin{array}{c}\text { Sarcopenia } \\
\quad(n=34)\end{array}$ & $\begin{array}{c}\text { Non- } \\
\text { sarcopenia } \\
(\mathrm{n}=23)\end{array}$ & P-value \\
\hline Age (years) & $77.5 \pm 7.9$ & $73.4 \pm 7.6$ & $0.041^{*}$ & $77.4 \pm 8.0$ & $80.0 \pm 9.5$ & 0.269 \\
\hline Height $(\mathrm{cm})$ & $161.3 \pm 5.4$ & $165.6 \pm 6.2$ & $0.004 *$ & $150.3 \pm 5.7$ & $153.9 \pm 5.2$ & $0.019 *$ \\
\hline Weight (kg) & $57.6 \pm 6.6$ & $65.6 \pm 7.8$ & $<0.001 *$ & $46.3 \pm 8.0$ & $56.6 \pm 7.9$ & $<0.001^{*}$ \\
\hline BMI $\left(\mathrm{kg} / \mathrm{m}^{2}\right)$ & $22.2 \pm 2.6$ & $24.0 \pm 3.0$ & $0.012^{*}$ & $20.6 \pm 4.0$ & $23.9 \pm 3.1$ & $0.001 *$ \\
\hline Care level $(1-7)^{\dagger}$ & $3(2-3)$ & $3(2-4)$ & 0.659 & $3(2-4)$ & $2(1-3)$ & $0.011 * *$ \\
\hline Smoking history ${ }^{\dagger \dagger}$ & 28 & 12 & 0.411 & 1 & 0 & 1.000 \\
\hline \multicolumn{7}{|l|}{ Sarcopenia assessment } \\
\hline Grip strength (kg) & $22.9 \pm 5.6$ & $31.0 \pm 5.0$ & $<0.001 *$ & $15.6 \pm 4.8$ & $19.0 \pm 4.6$ & $0.010^{*}$ \\
\hline Usual gait $(\mathrm{m} / \mathrm{s})$ & $0.64 \pm 0.27$ & $0.77 \pm 0.34$ & 0.085 & $0.52 \pm 0.24$ & $0.68 \pm 0.39$ & 0.084 \\
\hline $\operatorname{SMI}\left(\mathrm{kg} / \mathrm{m}^{2}\right)$ & $6.07 \pm 0.57$ & $7.40 \pm 0.78$ & $<0.001^{*}$ & $5.13 \pm 0.44$ & $6.28 \pm 0.48$ & $<0.001^{*}$ \\
\hline $\begin{array}{l}\text { Trunk muscle mass per } \\
\text { square height }\left(\mathrm{kg} / \mathrm{m}^{2}\right)\end{array}$ & $6.99 \pm 0.75$ & $7.84 \pm 0.90$ & $<0.001^{*}$ & $6.29 \pm 0.57$ & $6.88 \pm 0.62$ & $0.001^{*}$ \\
\hline \multicolumn{7}{|l|}{ Respiratory function } \\
\hline $\mathrm{FVC}(\mathrm{L})$ & $2.23 \pm 0.68$ & $2.52 \pm 0.64$ & 0.096 & $1.55 \pm 0.40$ & $1.66 \pm 0.43$ & 0.352 \\
\hline FEV1.0 (L) & $1.88 \pm 0.56$ & $2.10 \pm 0.59$ & 0.120 & $1.35 \pm 0.35$ & $1.41 \pm 0.37$ & 0.561 \\
\hline FEV1.0\% $(\%)$ & $84.5 \pm 7.4$ & $83.8 \pm 8.5$ & 0.736 & $87.2 \pm 6.9$ & $84.8 \pm 7.1$ & 0.197 \\
\hline PEFR (L/s) & $4.20 \pm 1.48$ & $4.69 \pm 1.90$ & 0.244 & $2.91 \pm 1.03$ & $3.56 \pm 1.26$ & $0.038^{*}$ \\
\hline \multicolumn{7}{|l|}{ Respiratory muscle strength } \\
\hline $\operatorname{MEP}\left(\mathrm{cmH}_{2} \mathrm{O}\right)$ & $52.8 \pm 18.4$ & $67.7 \pm 23.9$ & $0.006^{*}$ & $36.2 \pm 12.6$ & $45.7 \pm 14.6$ & $0.011^{*}$ \\
\hline $\mathrm{MIP}\left(\mathrm{cmH}_{2} \mathrm{O}\right)$ & $38.3 \pm 20.6$ & $43.6 \pm 16.2$ & 0.282 & $29.8 \pm 10.8$ & $33.0 \pm 18.2$ & 0.464 \\
\hline \multicolumn{7}{|l|}{ Morbidity } \\
\hline Hypertension & 17 & 10 & 1.000 & 15 & 10 & 1.000 \\
\hline Cerebrovascular dis & 23 & 16 & 0.317 & 14 & 7 & 0.576 \\
\hline Orthopedic dis & 22 & 9 & 0.317 & 25 & 17 & 1.000 \\
\hline Cancer & 11 & 4 & 0.545 & 5 & 4 & 1.000 \\
\hline Intractable neurological dis & 9 & 4 & 0.757 & 5 & 1 & 0.385 \\
\hline
\end{tabular}

2

3

4

5

6

7

$* \mathrm{P}<0.05$ for unpaired $t$ test.

$* * \mathrm{P}<0.05$ for Wilcoxon rank-sum test.

${ }^{\dagger}$ Median (25th percentile-75th percentile).

†W

BMI, body mass index; SMI, skeletal muscle mass index; FVC, forced vital capacity; FEV1.0, forced expiratory volume in 1 second; PEFR, peak expiratory flow rate; MEP, maximal expiratory pressure; MIP, maximal inspiratory pressure; dis, disease. 


\section{Table 2 (on next page)}

Table 2. Association between sarcopenia and respiratory parameters based on binomial logistic regression analysis

$\mathrm{Cl}$, confidence interval; MEP, maximal expiratory pressure; $\mathrm{BMI}$, body mass index. Dependent variables: Non-sarcopenia $=0$, sarcopenia $=1$. Independent variables: $M e n=0$, women $=1$;

without morbidity $=0$, with morbidity $=1$. Nagelkerke $R^{2}=0.281$. 
1 Table 2. Association between sarcopenia and respiratory parameters based on 2 binomial logistic regression analysis

\begin{tabular}{lllll}
\hline & $\beta$ & P-value & Odds ratio & $95 \% \mathrm{CI}$ \\
\hline $\mathrm{MEP}\left(\mathrm{cmH}_{2} \mathrm{O}\right)$ & -0.028 & 0.034 & 0.973 & $0.948-0.998$ \\
$\mathrm{Sex}$ & -0.988 & 0.056 & 0.373 & $0.135-1.028$ \\
Age $($ years $)$ & -0.004 & 0.868 & 0.996 & $0.945-1.049$ \\
$\mathrm{BMI}\left(\mathrm{kg} / \mathrm{m}^{2}\right)$ & -0.239 & 0.002 & 0.788 & $0.677-0.916$ \\
Certification $(1-7)$ & 0.323 & 0.052 & 1.381 & $0.998-1.911$ \\
Hypertension & 0.275 & 0.540 & 1.316 & $0.547-3.171$ \\
Cerebrovascular disease & -0.116 & 0.819 & 0.890 & $0.330-2.400$ \\
Orthopedic disease & 0.248 & 0.608 & 1.282 & $0.496-3.310$ \\
Cancer & -0.243 & 0.655 & 0.784 & $0.270-2.278$ \\
Intractable neurological disease & 0.037 & 0.959 & 1.037 & $0.258-4.167$ \\
\hline
\end{tabular}

3 CI, confidence interval; MEP, maximal expiratory pressure; BMI, body mass index.

4 Dependent variables: Non-sarcopenia $=0$, sarcopenia $=1$.

5 Independent variables: Men=0, women=1; without morbidity $=0$, with morbidity $=1$.

6 Nagelkerke $\mathrm{R}^{2}=0.281$. 


\section{Table 3 (on next page)}

Table 3. Partial correlation of MEP and MIP with component factors

Controlling for sex and age. BMI, body mass index; SMI, skeletal muscle mass index; MEP, maximal expiratory pressure; MIP, maximal inspiratory pressure. 
1 Table 3. Partial correlation of MEP and MIP with component factors

\begin{tabular}{lllll}
\hline & MEP & P-value & MIP & P-value \\
\hline Height $(\mathrm{cm})$ & 0.116 & 0.203 & 0.019 & 0.834 \\
Weight $(\mathrm{kg})$ & 0.370 & $<0.001$ & 0.204 & 0.024 \\
BMI $\left(\mathrm{kg} / \mathrm{m}^{2}\right)$ & 0.315 & $<0.001$ & 0.199 & 0.028 \\
Grip strength $(\mathrm{kg})$ & 0.263 & 0.003 & 0.075 & 0.411 \\
Usual gait $(\mathrm{m} / \mathrm{s})$ & 0.238 & 0.008 & 0.124 & 0.173 \\
SMI $\left(\mathrm{kg} / \mathrm{m}^{2}\right)$ & 0.276 & 0.002 & 0.075 & 0.414 \\
Trunk muscle mass per height squared $\left(\mathrm{kg} / \mathrm{m}^{2}\right)$ & 0.315 & $<0.001$ & 0.098 & 0.281 \\
MEP $\left(\mathrm{cmH}_{2} \mathrm{O}\right)$ & - & - & 0.550 & $<0.001$ \\
MIP $\left(\mathrm{cmH}_{2} \mathrm{O}\right)$ & 0.550 & $<0.001$ & - & - \\
\hline
\end{tabular}

2 Controlling for sex and age.

3 BMI, body mass index; SMI, skeletal muscle mass index; MEP, maximal expiratory pressure;

4 MIP, maximal inspiratory pressure. 
Figure 1

Figure 1. Flowchart of participant recruitment

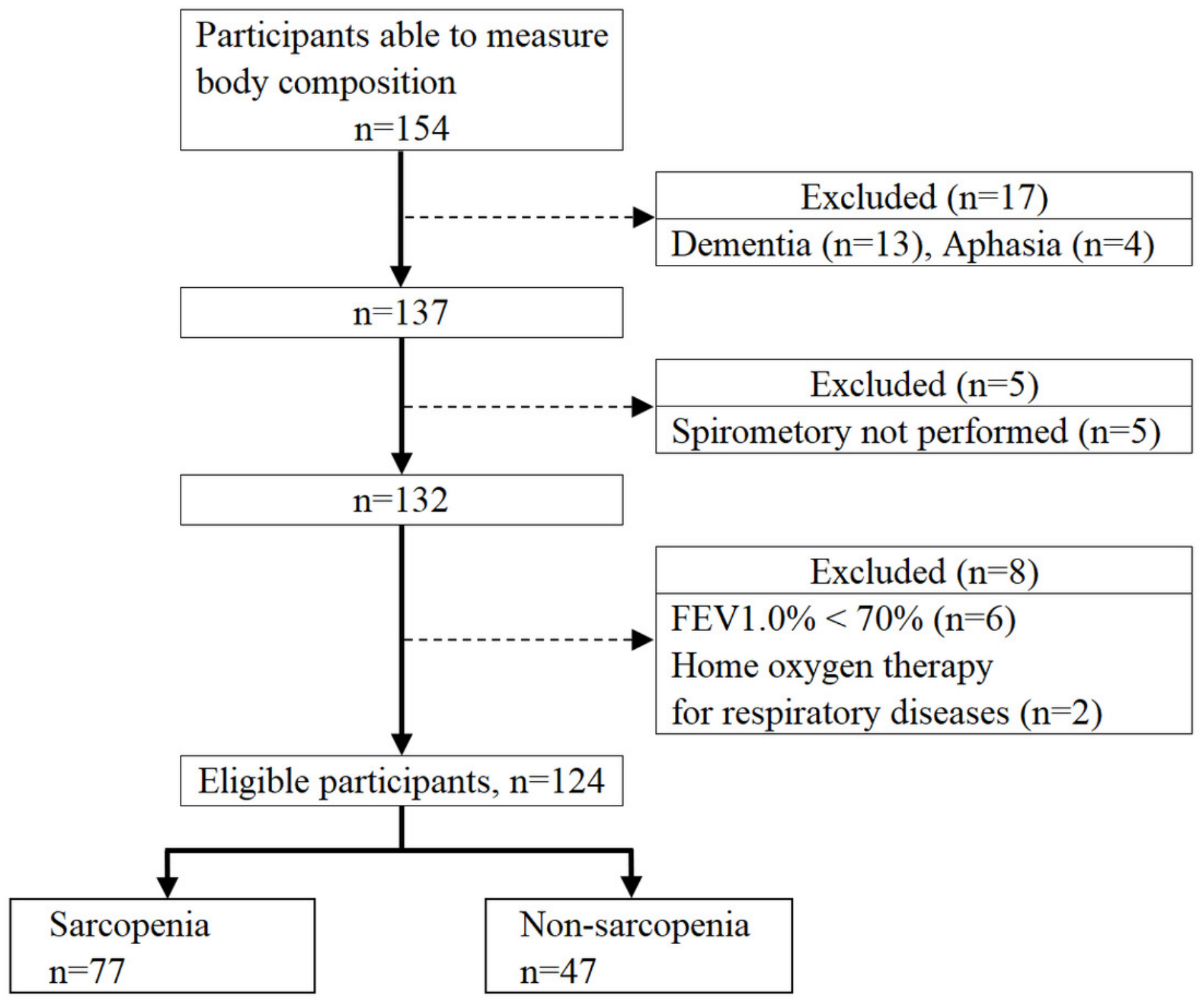


Figure 2

Figure 2. Receiver operating characteristic curve analysis for identifying sarcopenia via MEP

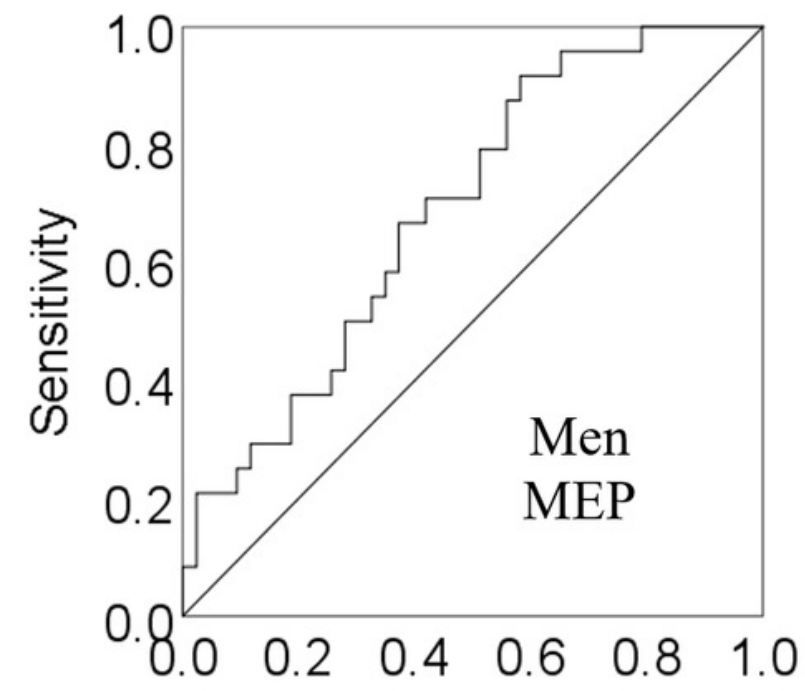

1 - specificity

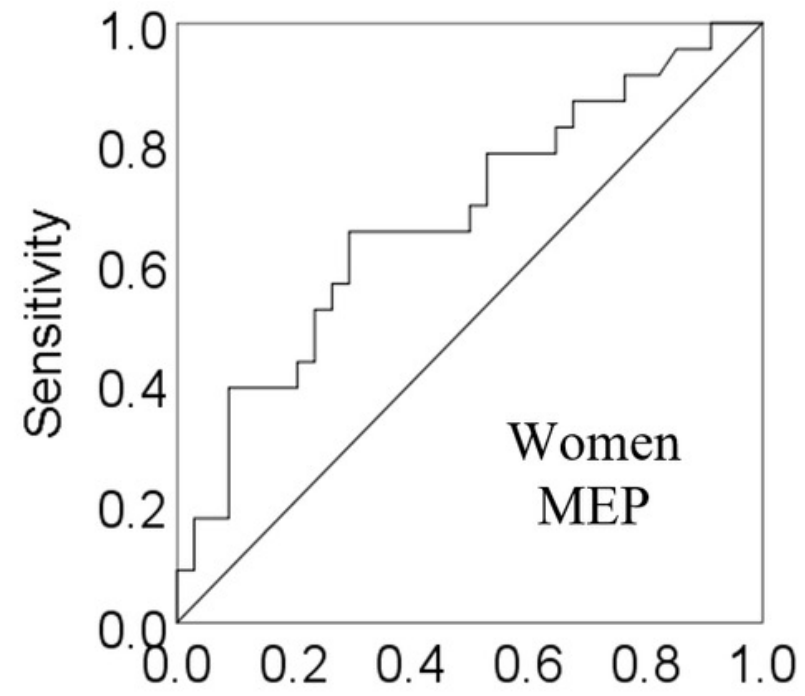

1 - specificity

\begin{tabular}{lcccccc}
\hline & Cut-off & Sensitivity & Specificity & AUC & $95 \% \mathrm{CI}$ & P value \\
\hline Men & $47.0 \mathrm{cmH}_{2} \mathrm{O}$ & $91.7 \%$ & $41.9 \%$ & 0.689 & $0.562-0.816$ & $0.011^{*}$ \\
Women & $40.9 \mathrm{cmH}_{2} \mathrm{O}$ & $65.2 \%$ & $70.6 \%$ & 0.677 & $0.533-0.821$ & $0.024^{*}$ \\
\hline
\end{tabular}

MEP, maximal expiratory pressure; AUC, area under the curve; CI, confidence interval.

$* \mathrm{P}<0.05$. 